\title{
ARCHEOLOGIE ET INTERPRETATION: POUR UNE SEMIOTIQUE DES OBJETS CULTURELS ARCHEOLOGY AND INTERPRETATION: FOR A SEMIOTIC OF CULTURAL OBJECTS
}

\author{
Bernard Lyonnet \\ Université de Franche-Comté \\ Laboratoire ELLIADD - Besançon \\ lyonnet.bernard@wanadoo.fr
}

Résumé: Dans le cadre du projet de sémiotique des cultures, le sémanticien François Rastier a précisé la notion d'objet culturel et proposé d'étendre les méthodes de la sémantique interprétative à des images et autres signes non-textuel. En parallèle, la nécessité de comprendre le sens d'objets symboliques révélés par l'archéologie est souvent exprimée et problématisée par les protohistoriens. Le croisement de ces deux domaines de recherche permet de poser les contours épistémologiques et méthodologiques d'une interprétation de ce type d'objets et d'en fournir une première illustration.

Mots clés: sémiotique des cultures, objet culturel, signes graphiques, interprétation, archéologie, protohistoire

Abstract: In the context of the semiotic of cultures project, the semanticist François Rastier has stated the notion of cultural object and has suggested to extend methods of interpretative semantics to images and other non-textual signs. Meanwhile, the necessity to understand the meaning of symbolic objects revealed by the archaeology is often expressed and problematized by protohistorians. The common ground of these two research areas allows us to set the epistemological and methodological outlines of an interpretation of such objects and to provide a first illustration.

Keywords: semiotic of cultures, cultural object, graphics signs, interpretation, archaeology, protohistory

François Rastier a initié un projet de sémiotique des cultures ${ }^{1}$ qui se veut une contribution à l'ensemble des sciences de la culture: «L'expression sciences de la culture est empruntée à Cassirer, pour qui elle s'oppose implicitement à sciences de la nature» [Rastier, 2002, p.4]. Une des bases de ce projet est sans doute dans le constat que:

«même promus au rang d'observables, les faits humains et sociaux restent le produit de constructions interprétatives. Aussi, les sciences de la culture sont les seules à pouvoir rendre compte du caractère sémiotique de l'univers humain» [Ibid. p.4].

Toute production culturelle peut être porteuse de sens et relève donc d'une sémiotique particulière au sein d'une sémiotique générale.

\footnotetext{
${ }^{1}$ Ce projet est exprimé dans un ouvrage collectif dirigé par Rastier et Bouquet : Une introduction aux sciences de la culture PUF, Paris, 2002.
} 
Dans un rappel des principes de sa démarche [Rastier, 2011, p. 24-25], il a ouvert une perspective qui confirme sa volonté d'aller au-delà de la seule refondation d'une linguistique unifiée. Il a ainsi proposé que:

(i) La problématique interprétative dépasse les textes et peut s'étendre à d'autres objets culturels, comme les images (susceptibles des mêmes méthodologies...).

(ii) La typologie et l'analyse des objets culturels exigent une réflexion anthropologique.

(iii) La sémiotique des objets culturels appelle enfin une réflexion sur l'ensemble des sciences de la culture.

Réflexion anthropologique, à partir des problématiques du langage, qui a fait aussi l'objet de propositions ${ }^{2}$.

Dans cet article nous nous proposons d'illustrer le premier de ces trois principes et d'ouvrir quelques perspectives, notamment en prenant en compte l'émergence d'une demande de cet ordre au sein des études protohistoriques en France. En Europe de l'ouest, la Protohistoire se pose comme une période charnière, associées surtout aux âges des métaux, caractérisée par l'apparition de témoignages écrits, souvent laconiques, donc insuffisants pour produire une relation détaillée d'évènements, de croyances ou doctrines. Ces témoignages écrits relèvent, pour les régions associées à l'aire culturelle celtique, soit d'une épigraphie indigène [Lambert, 2003, p 21], soit de récits ou de commentaires rédigés par les érudits des peuples voisins utilisant l'écriture. Outre le caractère incomplet de cette épigraphie, les textes historiques et ethnographiques sont le fait de conquérants Grecs puis Romains ayant souvent traité avec mépris les «barbares». Toutefois ces langues celtiques antiques, parlées notamment par les Gaulois, se sont conservées jusqu'à nos jours dans les îles britanniques où elles ont produit une abondante littérature épique, historique et juridique. En parallèle, les signes graphiques de l'art celtique et leurs motifs récurrents sont attestés depuis la fin du premier âge du fer (vers 500 av. n. è.) jusqu'au haut Moyen-âge insulaire, soit sur plus d’un millénaire.

\footnotetext{
2 Voir Rastier 2002, chapitre 14 : «Anthropologie linguistique et sémiotique des cultures », p. 243268.
} 
Pour illustrer une sémiotique de ces «autres objets culturels», les exemples que nous pouvons tirer de cette protohistoire ont un intérêt, sinon universel, au moins transposable à d'autres situations historiques et culturelles. Notamment, du fait du contexte colonial de la production des premiers textes archivés, par la comparaison qui pourrait être faite avec la situation des cultures précolombiennes de l'Amérique du sud confrontées à la conquête européenne.

Dans le cadre d'une thèse de doctorat [Lyonnet 2018], nous avons utilisé les outils de la sémantique interprétative pour renouveler l'approche de corpus irlandais médiévaux. Nous avons décrit des formes sémantiques pouvant servir de modèles pour des comparaisons avec des faits protohistoriques. Cependant seules quelques perspectives ont été ouvertes sur la possibilité d'interpréter certains objets de cet art celtique.

Nous nous proposons ici de présenter un aspect de cette problématique de l'interprétation d'objet artistiques dits sans paroles ${ }^{3}$, parce que non-commentés par des discours explicites contemporains. Tout d'abord en soulignant sa légitimité par l'émergence de questions qui s'expriment de façon de plus en plus pressante dans les débats des archéologues, ensuite en rappelant les propositions de François Rastier pour définir et décrire un «objet culturel». Nous terminerons par l'illustration de ces propositions méthodologiques à travers quelques exemples ouvrant la possibilité d'une description sémiotique et de modélisations sémantiques d'un motif graphique de l'âge du fer.

\section{1- L'émergence d'une archéo-sémiotique}

Au cœur même des découvertes archéologiques les plus matérielles surgit la question du sens et même d'un sens symbolique et/ou métaphorique : un tertre funéraire protohistorique n'est pas réductible à une masse de terre et de pierres explicable par les seules lois de la physique, ni même par les seules règles de l'économie et de la sociologie pour autant qu'elles soient elles-mêmes observables à des millénaires de distance. Il est un produit culturel et, à ce titre, un signe chargé de sens. Il en va ainsi des objets qu'il contient et qui n'y ont sans doute pas été

\footnotetext{
3 Par allusion au titre de l'ouvrage de l'archéologue Jean Abélanet, Signes sans paroles, Hachette, 1986.
} 
déposés par hasard, d'autant qu'ils peuvent porter la trace d'une iconographie dont les protohistoriens débattent pour savoir si elle a seulement une fonction décorative ou une fonction signifiante.

Dans le courant des années 2000, une série de recherches sur l'art protohistorique ont ouvert un dialogue avec la sémiotique. L'une des plus emblématiques est sans doute celle de l'anthropologue Alain Testart ${ }^{4}$, dans son ouvrage consacré au site néolithique anatolien de Çatal Höyük et à son iconographie. En introduction de son deuxième chapitre, il a posé la question d'une interprétation d'images anciennes consistant, dans un premier temps, à distinguer la part de la «représentation iconographique» de celle à «teneur religieuse» [Testart, 2010, p. 43]. Sur la base d'une interprétation de la «Vierge dorée» de la cathédrale d'Amiens, il a construit un modèle d'analyse pour lequel il a utilisé le vocabulaire de la sémiologie saussurienne, tout en le réinterprétant et sans produire de référence bibliographique à des auteurs représentatifs de ce courant [Ibid., p. 45-47] $]^{5}$.

Il y est donc question de sémantique, de signifiant et de signifié, de signe et même de sémiotique, un recours qui exprime une volonté de faire de la sémiotique dans le cadre d'une recherche associant protohistoire et ethnologie. Mais, tandis que le signifiant est associé à la dimension matérielle du signe «la statue en tant que chose matérielle», le signifié est renvoyé dans d'autres sphères: religieuse et symbolique. Cela semble entériner une conception dualiste du couple saussurien signifiant/signifié sans que le rapport entre les deux soit explicité ni même problématisé. Ici l'interprétation est en fait conventionnelle parce que c'est la doctrine chrétienne qui dicte le sens, notamment les sèmes /royauté céleste/ et /pudeur + modestie/. Ce sont les contraintes culturelles, celles de la doxa chrétienne, qui permettent d'identifier le motif de la /Vierge à l'enfant/ et les sèmes qui lui sont associés de façon récurrente au sein de ce contexte culturel.

L'intention interprétative de Testart ne porte pas tant sur le passage du signifiant au signifié, que sur la classification des signifiés entre le «religieux» et le

\footnotetext{
${ }^{4}$ Alain Testart, décédé le 2/09/2013, était directeur de recherche au CNRS et membre du Laboratoire d'anthropologie sociale du collège de France.

5 Pour le détail des citations et de l'analyse de ce passage, voir Lyonnet 2018 [en ligne], La sémiologie comme recours, p. 38-43.
} 
«symbolique», et accessoirement le «sémiotique et/ou artistique». Cela répond au problème initial de l'archéologue qui se trouve face à un vestige artistique et qui cherche à identifier sa finalité, son «intention», religieuse ou simplement esthétique. Cela implique au surplus des présupposés quant aux pratiques sociales des peuples anciens: car nous n'avons aucune certitude sur les éventuelles distinctions entre l'art et la religion que pouvaient faire les peuples néolithiques, pas même sur leur conception de ce que nous appelons «religion» après deux millénaires de christianisme.

Des archéologues spécialistes de l'âge du fer ont aussi emprunté des notions à la sémiotique. Ainsi André Rapin propose de mettre en œuvre une sémiologie des images de l'âge du fer. Il la situe «dans la suite logique des recherches typochronologiques», constituant «un préalable indispensable au travail des analystes de l'art» en permettant «l'identification objective des signes, de leur assemblage, de la construction des motifs, de leur combinatoire, de leur syntaxe etc.». Cela pour finalement distinguer entre décor et signe [Rapin, 2003, p. 49-50], ce qui revient encore à séparer l'artistique du symbolique.

Dans la même publication, Nathalie Ginoux mettait en cause les approches «s'intéressant exclusivement aux contenus idéologiques, symboliques ou religieux des compositions» de l'art celtique en posant que «l'aptitude de l'approche langagière à dégager du sens reste à démontrer». Elle voulait montrer que «l'approche langagière est plus métaphorique qu'opératoire, et qu'elle aboutit dans le meilleur des cas, à occulter de bonnes études formelles par des stéréotypes et dans le pire des cas à les entraver, en faussant les données descriptives de base». Elle appelle «métaphore langagière» la démarche qui consiste à «substituer aux méthodes propres à l'histoire de l'art les outils de la linguistique» [Ginoux, 2003, p. 259-260].

Plus qu'une réaction corporatiste face à la multiplication des emprunts à une sémiologie issue des recherches linguistiques, la mise en cause de Nathalie Ginoux pose des questions qui intéressent directement notre démarche, puisque, d'une certaine façon, elle va jusqu'à remettre en cause la possibilité même de l'existence d'un signifié lorsqu'elle produit deux schémas distinguant les étapes d'identification, d'analyse puis d'interprétation, en proposant de réduire la visée de 
cette dernière à une «nouvelle définition du style» en termes de «technostylistique et techno-économique» par opposition à la visée langagière d'un «système symbolique du discours» et l'interprétation iconologique d'une «symbolique des productions visuelles» [Ginoux, 2003, fig. 5 et 6, p. 268-269].

En conclusion de son article elle propose une «approche formaliste», à ses yeux c'est «l'interaction entre les éléments formels qui produit du sens, sans qu'il soit nécessaire de substituer au concept de composition celui de syntagme» [Ginoux, 2003, p. 268]. Elle tire argument, en note, de Paul Klee qui avait «isolé le visuel (pictural) du littéraire (...) pour fonder le sens d'une pensée visuelle (...) indépendamment de toute connotation littéraire» [Ginoux, 2003, p. 271, n. 13]. Cette référence réduit les signes aux «points, lignes, compositions» et use du terme «sens» sans que ce qu'il pourrait recouvrir soit précisé. Ce qui nous permet un parallèle avec l'exemple chrétien de Testart, puisque dans les deux cas ces chercheurs fondent leur modèle d'interprétation sur une doctrine connue, mais anachronique: rien ne dit que la pensée chrétienne soit éclairante pour comprendre l'art néolithique de Çatal Höyük, ni celle de Klee pour l'art celtique réputé «abstrait» ${ }^{6}$. Nathalie Ginoux aboutit à la sentence que «l'archéologie peut se passer de l'axe linguistique pour aborder ses sources iconographiques» [Ginoux, 2003, p. 270].

Or cet article de Nathalie Ginoux conclut les actes d'un colloque ouvert par l'introduction d'Olivier Buchsenschutz, qui posait la question titre de la «signification des images dans le monde celtique»où il affirmait la nécessité d'analyser un «langage de signes» [Buchsenschutz, 2003, p. 8]. Buchsenschutz qui concluait, dans son étude sur la «morphologie des vases de La Tène», à propos de la forme des céramiques que: «nous espérons avoir démontré que ces types de céramiques n'avaient pas de raison d'être strictement fonctionnelle ou technique»

\footnotetext{
${ }^{6}$ La référence à Klee semble entériner le recours au cliché d'un "art celtique abstrait », alors que les analyses de ceux qui le décrivent évoquent plutôt un art, certes non réaliste, mais dans lequel des figurations humaines, animales et végétales sont identifiables, associées à des figures géométriques récurrentes comme la triscèle (triple spirale) ou l'esse (double spirale). Sa principale caractéristique résidant dès lors dans un enchaînement complexe de ces diverses figures donnant une impression de métamorphose perpétuelle, cf. Kruta, 2000, p. 729, art. «Métamorphose plastique».
} 
pour justifier leur interprétation en termes de «signification» [Buchsenschutz, 2003, p. 89].

Laurent Olivier précise par ailleurs que:

\begin{abstract}
«ces codes protohistoriques étant fondamentalement différents de ceux transmis par la tradition naturaliste de l'art occidental, les chercheurs du XIXe siècle en ont inconsciemment déduit que ceux-ci étaient primaires», ce qui a eu «pour conséquence de repousser spontanément les figurations de l'art de la Tène aux confins de l'ornemental (...). Ces interprétations témoignent surtout d'une position idéologique qui place (...) les figurations stylisées ou géométriques des sociétés 'primitives' ou 'barbares' à un stade inférieur, du point de vue du développement intellectuel des civilisations, à celui des figurations naturalistes des sociétés classiques» [Olivier 2014, p. 39].
\end{abstract}

Cette différence de point de vue entre des protohistoriens constitue sans doute la matière d'un débat fécond, mais elle montre surtout que ce que vise Nathalie Ginoux avec sa dénonciation de la «métaphore langagière» ce n'est pas tant le travail des linguistes et des sémiologues, que d'historiens de l'art faisant des emprunts à ces disciplines. La question est celle de la difficulté de l'emprunt de notions à d'autres disciplines, et elle nous semble légitimer la nécessité d'une recherche faisant le chemin inverse: c'est-à-dire un parcours de sémiologue dans un corpus de documents protohistoriques.

\title{
2-Sémiotique des objets culturels et perspectives anthropologiques
}

Un point essentiel de l'apport de l'œuvre de Rastier à l'anthropologie et à la compréhension du phénomène sémiotique consiste à le poser comme «environnement humain» [Rastier 2014, p.439]. En précisant l'idée que les signes et le langage ne sont pas «les instruments de la pensée ni l'expression de comptesrendus de perceptions», il rappelle le rejet fondateur de la réduction du sens à la référence (ontologie) ou à des concepts produits par un esprit (métaphysique) tel que le principe saussurien de différence permet de les dépasser. Mais de façon encore plus nouvelle et qui signe son apport fondamental: l'esprit-pensée n'est plus placé entre le monde (référent) et le signe (expression) selon la tradition aristotélicienne, faisant de ce dernier le simple instrument d'une pensée lui préexistant a priori, mais c'est le signe qui occupe la position centrale. Ainsi l'être humain, conditionné par l'apprentissage plus que par l'hérédité biologique, tout au moins au niveau du cerveau, et particulièrement par l'apprentissage du langage, vit 
dans un univers de signes à travers lesquels il perçoit le monde et il forme sa pensée.

Le modèle triadique classique de la signification linguistique posait l'activité conceptuelle au centre, sur le modèle d'un esprit pensant autonome:

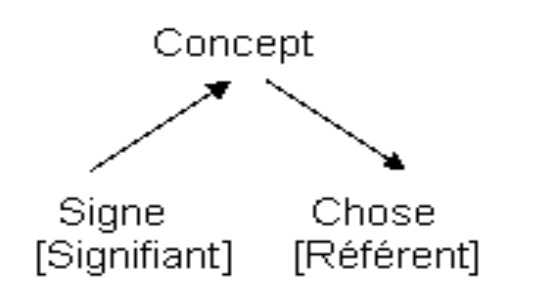

Le signe, «réduit à sa simple expression (signifiant), renvoie à un objet (un référent) par la médiation d'un concept» [Duteil-Mougel, 2004].

La rupture proposée par Rastier a pour conséquence une réorganisation de la triade sémiotique qui peut être présentée ainsi:

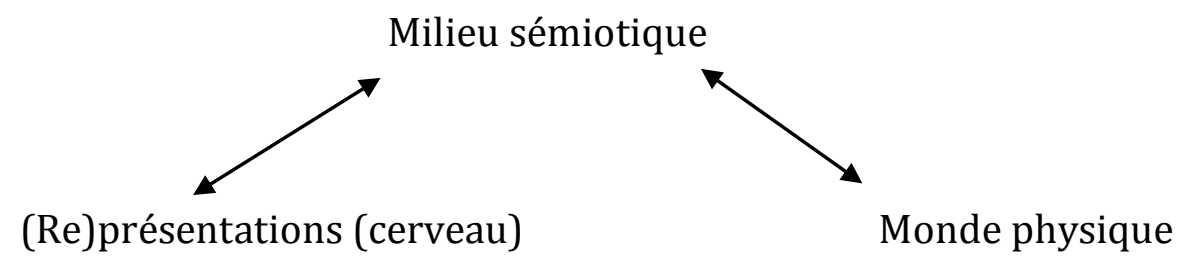

Ici le signe, dans son milieu sémiotique, est dans une position médiane, mais en interaction avec les autres signes ainsi qu'avec le monde et le cerveau. Ce dernier étant en quelque sorte contraint par le milieu sémiotique, donc la culture héritée, dans sa perception et sa conception du monde. Ce milieu sémiotique, c'està-dire l'ensemble des signes et des performances, interagit avec d'une part le monde physique, dans un sens en lui empruntant des supports (phonèmes, images) mais aussi en conditionnant la perception que nous en avons, selon notre héritage culturel et linguistique. D’autre part le milieu sémiotique interagit avec le cerveau, très probable siège de l'activité neuronale liée à l'activité linguistique, en 
lui empruntant d'un côté les éléments mémorisés ${ }^{7}$ et en lui fournissant les signifiés et signifiants élaborés et appris dans la pratique sémiotique.

Les signes, conçus sur la base du couple signifiant/signifié interagissent entre eux selon le principe différentiel. Toutefois ils ne sont pas complètement indépendants de la pratique sociale au sein de laquelle ils sont produits. C'est pour décrire cette interaction entre la pratique sociale et les signes, que Rastier propose un modèle de l'objet culturel intégrant une double dualité [Rastier 2011, p. 54]:

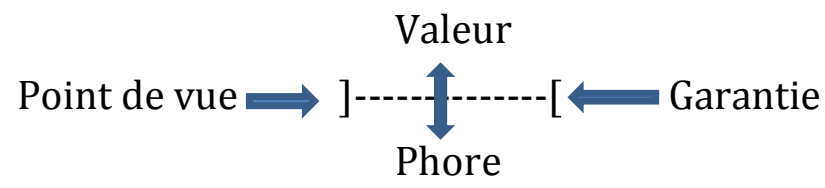

Phore et Valeur «désignent respectivement les expressions et les contenus pour tout système de signes» [Ibid., p. 53], c'est-à-dire le signifiant et le signifié. Cette dualité «se trouve sous la rection d'une dualité de rang supérieur entre le Point de vue et la Garantie». Le premier n'est pas un «simple point d'observation: il est déterminé par une pratique et un agent individuel ou collectif», tandis que la seconde «est l'instance de validation qui fonde l'évaluation des 'données': cette instance est une norme sociale qui peut être juridique, scientifique, religieuse ou simplement endoxale» [Ibid., p. 54].

Ainsi le contenu (Valeur) d'une expression (Phore) ne peut être interprété sans le contexte de l'ensemble des signes associés (par exemple un texte pour des lexèmes) et sans le Point de vue qui détermine la pratique, ni la Garantie qui assure l'authenticité de l'objet culturel et des normes qui conditionnent sa production. Et c'est bien tout le problème de l'interprétation d'un signe ancien dont le contexte et la pratique sociale sont peu documentés.

\footnotetext{
${ }^{7}$ Les psychologues cognitivistes spécialistes de la mémoire parlent d'une mémoire sémantique qui est le socle de la mémoire de longue durée. S'ils intègrent la notion de traits sémantiques, leurs modèles classificatoires sont issus de l'informatique et ils hésitent entre des systèmes de stockage arborescents ou en «liste de traits », tout en admettant que les " jugements sémantiques », en fait les requêtes au sein de la mémoire sémantique, se fassent par comparaison. Voir Alain Lieury, Psychologie de la mémoire, 2005, p.125. Il pourrait être intéressant de dépasser la simplicité des modèles classificatoires de l'Intelligence Artificielle en exploitant les modèles plus complexes mais textuellement attestés produits par la SI.
} 
Dans le cas des vestiges archéologiques, le contexte matériel fournit toutefois une série d'indices. Le contexte funéraire ou cultuel d'un dépôt indique une pratique de type religieux, et la répétition des mêmes offrandes, composées des mêmes types d'objets, dans des contextes analogues indique le caractère endoxal, donc normatif, de cette pratique. L'accumulation de données comparables permet de constituer des corpus d'objets culturels susceptibles d'un traitement sémiotique dans la mesure où ces pratiques les désignent comme signes. Cette répétition même de gestes analogues, devenant par-là rituels, ou de motifs graphiques signe leur dimension culturelle. Parce qu'elle est partagée, la culture est répétition et reformulation : une création première n'a de valeur culturelle qu'à partir du moment où elle est reprise, reproduite et transmise. Ce qui implique, outre la répétition dans son aspect quantitatif qui signale sa portée en synchronie, la prise en compte d'une dimension temporelle: un signe graphique comme la triple spirale (triscèle) en contexte celtique ou une forme sémantique comme l'analogie $/$ arbre $=$ savoir $/{ }^{8}$ ont une dimension culturelle justement parce qu'ils ont été transmis sur plusieurs générations voire plusieurs siècles.

Dès lors, en l'absence d'une documentation ethnographique complète comme c'est le cas pour les objets culturels protohistoriques, deux solutions s'offrent pour l'interprétation. Tout d'abord prendre appui sur la description des images elles-mêmes, en faisant l'hypothèse que, hormis les cas d'écritures conventionnelles non-hiéroglyphiques, la matérialité des formes implique une relation analogique entre l'expression et son contenu. Ensuite prendre appui sur les éléments linguistiques connus pour une modélisation sémantique: pour la période de l'âge du fer, le lexique permet d'avoir accès à des signifiés accessibles et, entre les mots, à des différences qui structurent des embryons de taxèmes et domaines sémantiques; en complément, les dimensions qui structurent ces ensembles sémantiques appartiennent à une temporalité plus longue qui permet des comparaisons avec les sources textuelles de langues apparentées mais de périodes voisines. Ces dimensions constituent des normes, plus ou mois durables,

\footnotetext{
${ }^{8}$ Elle explique l'étymologie du mot celtique druide et se trouve reformulée et institutionalisée dans divers documents en Irlande médiévale et dans un temps long. Voir le chapitre 5 de notre thèse, Lyonnet, 2018.
} 
qui peuvent offrir quelques garanties quant aux choix sémantiques opérés lors de l'interprétation. Ceci, à condition de pratiquer une modélisation, permettant d'organiser et décrire des faits nouveaux ou mal connus, et pas une transposition arbitraire d'une période sur une autre [Lyonnet 2018, p. 97, p. 266, p. 287].

Dans la distinction entre la langue et la parole que Rastier reprend à Saussure, le sémanticien a introduit une notion de temporalité:

«Elles (les normes) peuvent être remaniées avec l'évolution des pratiques sociales où ils (les discours) prennent place, et elles évoluent donc dans une temporalité différente de celle de langue, faite de normes invétérées» [Rastier 2001, p. 154].

Ainsi les normes invétérées, c'est-à-dire qui se sont figées avec le temps, sont devenues des règles qui évoluent peu ou lentement, tandis que les normes sociales, qui pèsent sur les activités de discours et leurs genres, «peuvent être remaniées». Toutefois, même dans d'ordre paradigmatique des classes sémantiques, les taxèmes peuvent être remaniés au sein d'un domaine, en fonction du corpus de référence, tandis que ces domaines sont corrélés à une activité sociale culturellement plus stable, alors que les dimensions qui rendent visible le système de valeurs ont une plus grande inertie.

Ces parallèles suggèrent trois temporalités distinctes qui ne sont pas sans rappeler les trois temporalités braudéliennes [Lyonnet 2018, p. 95]:

\begin{tabular}{|l|l|l|l|l|l|}
\hline Temps long & Millénaire? & Langue & Règles & Dimensions & Valeurs \\
\hline $\begin{array}{l}\text { Temps } \\
\text { moyen }\end{array}$ & Siècle? & Discours & Normes sociales & Domaines & Institutions \\
\hline Temps court & Génération? & Textes & $\begin{array}{l}\text { Normes sociales } \\
\text { et/ou individuelles }\end{array}$ & Taxèmes & Activités \\
\hline
\end{tabular}

Cette idée de trois rythmes différents d'évolution des objets sémantiques et culturels pourrait avoir une certaine importance pour refonder épistémologiquement le comparatisme en diachronie. Par exemple au sein des études celtiques entre les faits gaulois et les faits irlandais qui leur sont postérieurs de quelques siècles. Ce qui aussi pourrait expliquer que des éléments structurés qui sont de l'ordre du système de valeur, comme par exemple la tripartition dumézilienne, puissent se transmettre dans la très longue durée tout en produisant, au niveau de l'expression (phore), d'infinies variations liées à des 
contextes culturels différenciés et en évolution constante. C’est la durabilité de ce type d'objets sémantiques qui confirme leur dimension culturelle et qui légitime leur utilisation comme modèles.

Ainsi, le milieu sémiotique est central dans l'activité humaine, entre le monde et le cerveau, et il contraint nos pensées et perceptions. Pour autant qu'ils soient partagés de façon conséquente et transmis durablement, les signes ont en outre une valeur culturelle et sont constitutifs d'une doxa. Ils s'intègrent à des pratiques sociales dont ils préservent une partie essentielle de la mémoire, dès lors que leur système de valeurs est descriptible [Lyonnet 2018, p. 101-103]. Ils sont donc un objet d'étude, historique et anthropologique, à part entière compte tenu du rôle qu'ils jouent dans les cultures et civilisations. Pour les périodes anciennes dont l'archive textuelle est fragmentaire, il est possible d'étayer l'interprétation de signes graphiques en procédant à des modélisations sémantiques s'appuyant à la fois sur les éléments connus de la langue contemporaine, et sur des modèles de comparaison pris dans des cultures linguistiquement apparentées, à condition que leur durabilité soit attestée.

\section{3-Sémiotique de quelques objets protohistoriques}

Dans une publication récente, issue d'un travail de thèse, l'historien

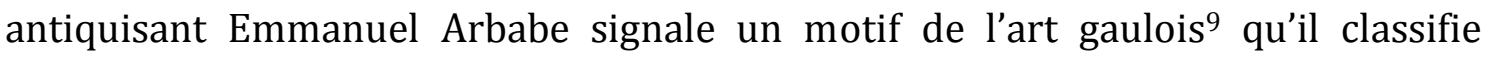
comme «un insigne du commandement militaire», après avoir listé de nombreuses attestations s'étalant sur «une période allant du VI ${ }^{\mathrm{e}}$ siècle à la fin du I ${ }^{\mathrm{er}}$ premier siècle av. n. è.». Ce motif «prend la forme de trois cercles ou boules disposés en triangle que l'on reconnaît sur de nombreux supports» [Arbabe 2017, p. 228-229]. L'auteur présente en outre une abondante iconographie en annexe permettant de visualiser ce motif [Ibid., p. 355-359]. Cette illustration intervient dans un passage où l'historien, sur la base d'une relecture très étayée des textes classiques dont la Guerre des Gaule de Jules César, distingue différentes fonctions du pouvoir

\footnotetext{
${ }^{9}$ Rappelons à toutes fins utiles que les Gaulois parlent une langue celtique attestée par l'épigraphie et sont désignés comme Celtes par plusieurs auteurs gréco-latins.
} 
politique très proches du modèle ternaire Galate et associant, de façon temporaire et éligible, un roi ou vergobret ${ }^{10}$ et un chef militaire à un druide [Ibid., p. 232].

Dans une logique référentielle propre aux historiens, Arbabe cherche à identifier la fonction pratique et sociale de ce motif à trois boules et en tire argument pour étayer l'existence, chez les Gaulois de l'indépendance, d'un rôle politique et militaire distinct de celui du vergobret.

Sans discuter cet aspect de la recherche historique constatons que, d'un point de vue sémiotique, nous pouvons nous interroger sur le sens de ce signe récurrent et durable constitué de trois cercles et/ou trois boules.

Un premier élément d'observation peut porter sur les associations syntagmatiques. Ainsi la présence de ce motif est fréquente sur des casques [Ibid., p. 357-359, fig. 11a-h,12]. Arbabe en retient deux du dépôt de Tintignac. L'un d'entre eux est surmonté de trois grands cercles soudés: «trois grands anneaux de $280 \mathrm{~mm}$ de diamètre chacun (...) disposés en triangle au-dessus du casque» [Maniquet 2008]. Dans le même dépôt, un casque de fer comprend «deux tôles circulaires ornées elles-mêmes d'un motif basé sur le triscèle (...) et une tôle triangulaire occupe la face antérieure. Cette dernière est ornée de trois nodosités disposées en triangle»[Ibid.]. Ce qui permet un rapprochement avec le casque d'Amfreville dont une bande dorée, décorée de triscèles (triples spirales) enlacés, occupe une zone juste en dessous du sommet, mais supérieure à une bande colorée d'émail rouge où le motif de la double spirale (esse) domine [Perrin 2011]. Constatons que les casques sont portés sur la tête, qu'ils protègent ou embellissent. Ce motif de trois boules ou cercles se trouve aussi sur des torques, de massifs colliers typiquement celtiques portés au ras du cou, donc marquant et soulignant la séparation entre la tête et le corps [Arbabe 2017, p. 356, fig. 10abc].

L'association récurrente de ce motif ternaire avec la tête semble confirmée par la fibule en bronze d'Oberwittighausen (Bade-Wurtenberg), datée du Ve siècle av. n. è. [Ibid., p. 355, fig. 9], que nous reproduisons ci-dessous:

10 Terme gaulois attesté par l'épigraphie et utilisé par César, cf. Arbabe 2017, p. 230-231. 


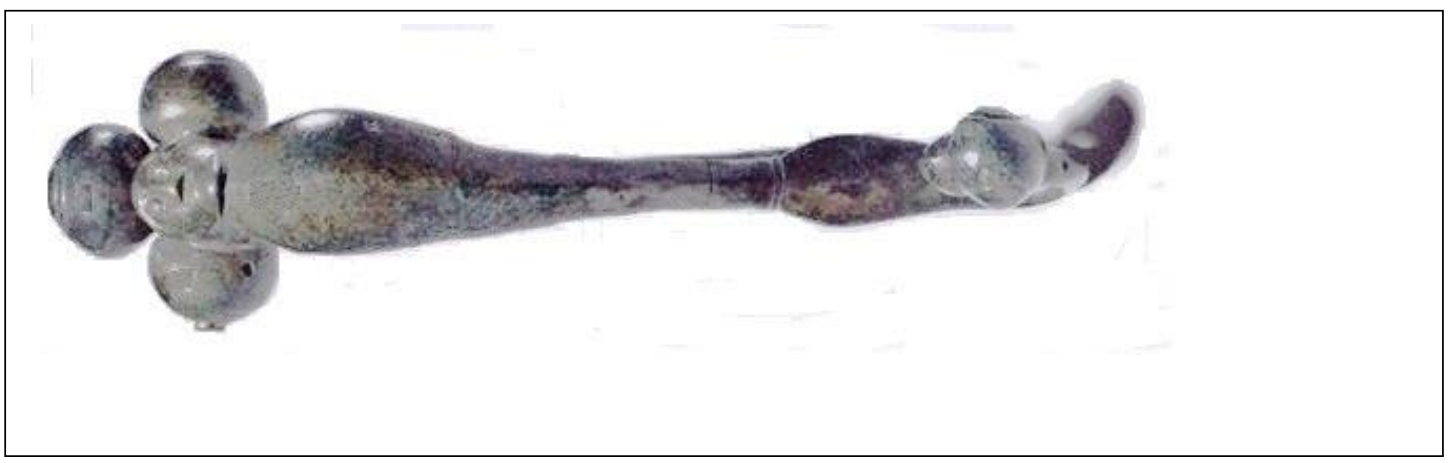

L'observation de cette fibule suggère une distinction entre la tête plus ses trois boules et le corps de la fibule, qui stylise un torse bombé formant une courbe inversée avec les jambes, l'ensemble ayant l'aspect d'une esse, autre motif récurrent de l'art celtique.

L'hypothèse d'une différence entre la tête associée à trois boules/cercles et le corps associé quant à lui avec la double spirale S est renforcée par sa variante sur un autre support et une autre figuration, en l'occurrence animale ${ }^{11}$. Il s'agit d'un vase peint de Clermont-Ferrand daté du IIe siècle av. n. è. [Guichard 2000, p. 36-37] :

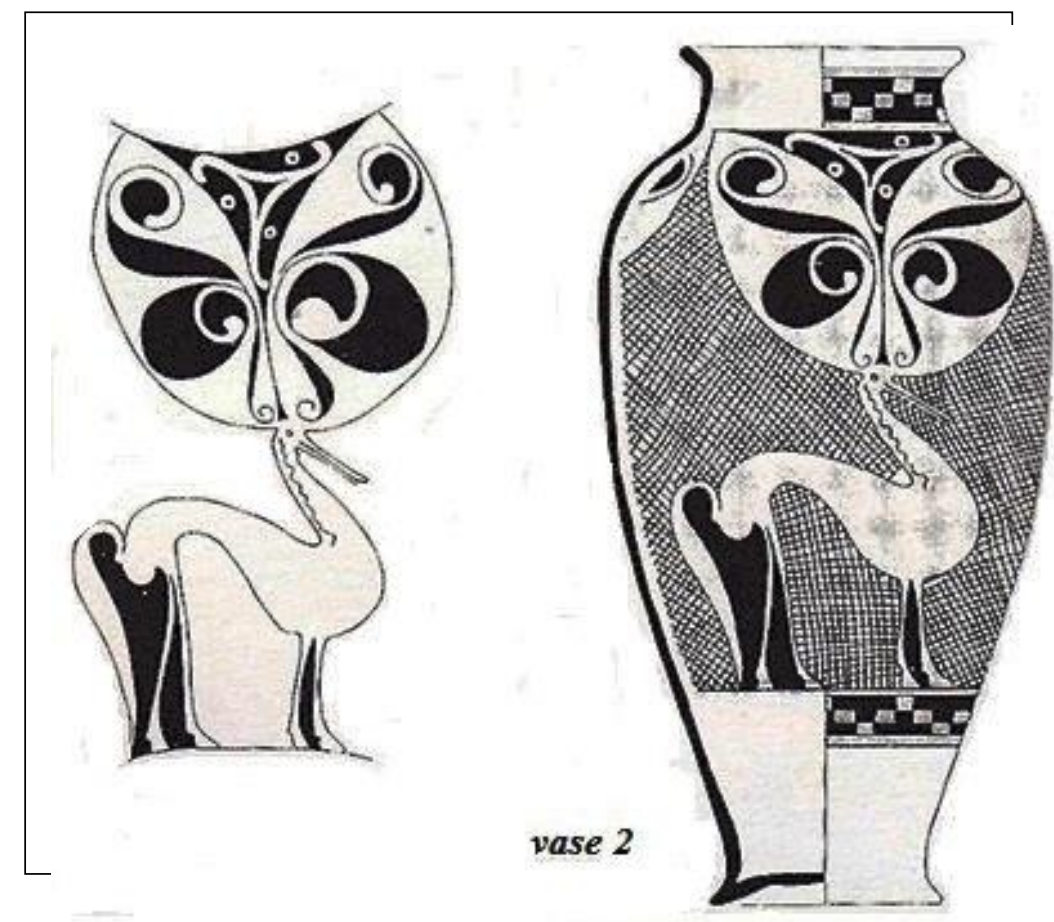

${ }^{11}$ Rappelons que les métamorphoses ou transferts figuratifs entre l'humain et l'animal sont une des caractéristiques de l'art celtique. Un dieu Cernunnos, représenté avec un corps humain et des ramures de cerf, est attesté. 
Ici le corps du cervidé prend la forme d'une double courbe en S horizontal, tandis que la ramure, elle-même formée de deux fois trois spirales en miroir, est explicitement associée à une triple spirale et un triangle composant le motif bien connu et récurrent du triscèle. De plus, trois cercles sont associés aux courbes du triscèle.

La répétition dans des associations syntagmatiques équivalentes de formes légèrement variantes (boule, cercle, spirale), mais systématiquement disposées en triangle, permet de les identifier à partir des sèmes communs /courbe + trois/.

Même si ce corpus doit être étoffé, nous pouvons déjà faire quelques constats au sujet de ce motif graphique :

- Nous sommes en présence d'un objet culturel, d'abord par sa répétition et transmission durable (au moins cinq siècles), ensuite par son inscription dans une ou des pratiques sociales, a minima artistiques, mais aussi très certainement rituelles et/ou à finalité politique selon l'hypothèse d'Arbabe.

- Les associations et oppositions signalent un procédé différentiel caractérisant un procès sémiotique: nous sommes bien en présence de signes symboliques car l'intention signifiante est exprimée par ces jeux de différences [Lyonnet 2018, p. 109].

- Ces jeux différentiels peuvent se reformuler ainsi: /tête + triple courbe $\neq$ corps $\pm \mathrm{S} /$, car la tête se voit effectivement ajouter la triple courbe tandis que le corps est représenté lui-même en double courbe. Ce qui constitue une forme sémantique pouvant être recherchée dans de nouvelles pièces enrichissant un corpus en construction.

Pour trouver une structure sémantique attestée en paroles et permettant d'expliciter et d'interpréter l'association /tête + triple courbe/, la tripartition du pouvoir décrite par Arbabe constitue déjà un solide candidat. La triade /chef militaire + druide + vergobret/ pourrait ainsi être associé à la tête symbolique d'une société ou cité celtique. En temps que norme garantissant l'interprétation, tant par les contemporains que par les observateurs tardifs que nous sommes, cette triade pourrait être rapprochée de celle découverte par Dumézil dans de très nombreuses traditions littéraires de la famille linguistique indo-européenne: un héros, un sorcier, un roi ${ }^{12}$. En contexte celtique elle est identifiable à l'association

${ }^{12}$ C'est le thème-titre de Mythe et épopée II, Quarto Gallimard 1995. 
/Lancelot + Merlin + Arthur/ des traditions médiévales inspirées de la matière de Bretagne. Ceci sans exclure à ce stade la possibilité de rapprochements avec d'autres triades bien attestées dans les textes celtiques.

À l'échelle de l'ensemble de la forme sémantique /tête + triple courbe $\neq$ corps $\pm S /$, nous pouvons tout d'abord rappeler la valorisation dont fait l'objet la tête en contexte celtique, notamment par des rites funéraires et religieux dont les auteurs classiques n'ont voulu retenir que l'aspect barbare. Toutefois l'archéologie confirme la conservation de têtes et leur valorisation dans des sanctuaires, ainsi d'ailleurs que la conservation de corps sans tête [Rousseau 2011].

Une autre piste est à envisager dans la possibilité d'une conception tripartite du corps humain, telle que l'a relevé Dumézil dans un récit irlandais médiéval ${ }^{13}$. Dans ce contexte culturel, trois grandes zones du corps correspondent aux trois fonctions thématiques et actantielles de la théorie dumézilienne, tel que nous pouvons la résumer dans ce tableau [Lyonnet 2018, p. 90]:

\begin{tabular}{|c|c|c|c|}
\hline Classification & $\begin{array}{c}\text { Première fonction - } \\
\mathbf{F}^{\circ} \mathbf{1}\end{array}$ & $\begin{array}{c}\text { Deuxième fonction }- \\
\mathbf{F}^{\circ} \mathbf{2}\end{array}$ & $\begin{array}{c}\text { Troisième fonction - } \\
\mathbf{F}^{\circ} \mathbf{3}\end{array}$ \\
\hline Thèmes & Savoir, pensée & Force, action & Fécondité, production \\
\hline Pratiques & $\begin{array}{c}\text { Droit, enseignement, } \\
\text { récit }\end{array}$ & Guerre, chevalerie & Agriculture, artisanat \\
\hline $\begin{array}{c}\text { Partie du } \\
\text { corps }\end{array}$ & Tête & Poitrine, cœur, bras & $\begin{array}{c}\text { Ventre, bas-ventre, } \\
\text { cuisses }\end{array}$ \\
\hline
\end{tabular}

Dans le cadre de ce modèle, la esse pourrait représenter les deux parties basses du corps, et la tête serait associée séparément à trois fonctions spirituelles ou intellectuelles pour lesquelles nous avons diverses possibilités de modélisations. Outre les trois pouvoirs d'Arbabe, nous pouvons signaler la triade antique /druide-barde-vate/ qui se prolonge dans la triade /druide-file-médecin/ de l'épopée irlandaise, sans exclure a priori les autres possibilités offertes par des triades divines.

\footnotetext{
${ }^{13}$ Voir Dumézil, 1995, Lugaid aux Raies Rouges, p.1019-1021.
} 


\section{Conclusion}

À ce stade, concernant l'interprétation d'objet culturels et signes celtiques de la protohistoire, nous n'avons fait que proposer quelques hypothèses. Toutefois, il s'agissait d'illustrer l'apport de la sémiotique des cultures à la réflexion historique en prenant en compte le milieu sémiotique comme constitutif d'une culture donnée. Puis, sur quelques exemples ayant effectivement une valeur culturelle par leurs diverses attestations et leur durée, de montrer la potentialité heuristique de la méthode sémantique.

En complément de la description que propose Rastier de l'objet culturel, nous avons souligné la nécessité de prendre en compte sa dimension temporelle durable, car il n'y a pas de culture sans transmission, et la nécessité de justifier le caractère sémiotique d'un tel objet par l'expression manifeste de procès différentiels observables. Ainsi des associations et oppositions de la forme sémantique /tête + triple courbe $\neq$ corps $\pm S /$, explicitement inscrites dans divers objets protohistoriques.

La méthode consiste alors :

1- À rassembler un corpus assurant la récurrence et la durée d'un motif graphique, ce qui fonde sa dimension culturelle.

2- $\mathrm{Au}$ niveau du contexte: à rassembler des indices sur les pratiques sociales auxquelles les supports du motif ont été intégrés (Point de vue).

3- Au niveau du signe (Valeur-Phore) : à décrire le signifiant dans ses rapports syntagmatiques pour observer les manifestations différentielles d'un système de valeur (opposition tête/corps) afin de faire des hypothèses sur les signifiés envisageables.

4- De chercher, par comparaison, des formes sémantiques attestées si possibles dans des éléments de langage (lexique, récits, etc.) et pouvant avoir eu valeur de normes ou de modèles (Garantie).

\section{Bibliographie}

Abélanet, Jean, Signes sans paroles, Hachette, Paris, 1986.

Arbabe, Emmanuel, La politique des Gaulois, éditions de la Sorbonne, Paris, 2017.

Buchsenschutz, Olivier et Bailly, Christophe, Recherche sur la morphologie des vases de La Tène, in Décors, images et signes de l'âge du fer européen, FERACF, Tour, 2003. Dumézil, Georges, Mythe et épopée I-II-III, Quarto Gallimard, Paris, 1995. 
Duteil-Mougel, Carine Introduction à la sémantique interprétative. Texto ! décembre 2004,

Ginoux, Nathalie, La forme: une question de fond dans l'expression non-figurative des sociétés sans texte, in Décors, images et signes de l'âge du fer européen, FERACF, Tour, 2003.

Guichard, Vincent, La céramique peinte, in Les Celtes et les arts du feu, Dossiers d'archéologie $n^{\circ} 258$, Dijon, 2000.

Kruta, Venceslas, Les Celtes, histoire et dictionnaire, coll. Bouquins, Laffont, Paris, 2000.

Lambert, Pierre-Yves, La langue gauloise, éd. Errance, Paris, 2003.

Lieury, Alain, Psychologie de la mémoire, 2005.

Lyonnet, Bernard, Pour une sémantique interprétative de l'archive celtique [En ligne], texto! volume XXIV - $\mathrm{n}^{\circ} 3$ (2018), URL : http://www.revuetexto.net/index.php?id=4112.

Maniquet, Christophe, Le dépôt cultuel du sanctuaire gaulois de Tintignac à Naves, Gallia 65, 2008.

Olivier, Laurent, Les codes de représentation visuelle dans l'art celtique ancien, in Celtic Art in Europe, Oxbow Books, Oxford, 2014.

Perrin, Franck, Symboliques du corail rouge, in Symboles celtiques, L'Archéologie $\mathrm{n}^{\circ}$ 113, Les Albres, 2011.

Rapin, André, Les analyses sémiologiques de l'image : l'iconographie du deuxième âge $d u$ fer, in Décors, images et signes de l'âge du fer européen, FERACF, Tour, 2003.

Rastier, François, Arts et sciences du texte, PUF, Paris, 2001.

Rastier, François, Pluridisciplinarité et sciences de la culture, in Introduction aux sciences de la culture, PUF, Paris, 2002.

Rastier, François, La mesure et le grain, Honoré Champion, Paris, 2011.

Rastier, François, La sémantique interprétative et les textes, in Documents, textes, œuvres, perspectives sémiotiques, Actes du colloque de Cerisy-la-Salle (juillet 2012), Presse Universitaires de Rennes, Rennes, 2014.

Rousseau, Élisabeth, Le symbole de la tête humaine, in Symboles celtiques, L'Archéologie ${ }^{\circ}$ 113, Les Albres, 2011.

Testart, Alain, La Déesse et le Grain, éd. Errance, Paris, 2010. 